\title{
ENVIRONMENTAL EFFECTS IN FREQUENCY SYNTHESIZERS FOR PASSIVE FREQUENCY STANDARDS
}

\author{
J. F. Garcia Nava\#, F. L. Walls*, J. H. Shirley*, W. D. Lee*, and M. C. Delgado Aramburo* \\ \# Centro Nacional de Metrologia, km 4.5 Carr, A Los Cues, C. P. 76900, Queretaro, Mexico \\ * National Institute of Standards and Technology, 325 Broadway Boulder, CO 80303, USA
}

\begin{abstract}
This paper reviews the environmental effects in synthesizers designed to support a frequency stability of $10^{-13} \tau^{-1 / 2}$ in short term and $10^{-17}$ in the long term. Specifically we consider the effects of temperature, pulling by spurious spectral lines, vibration effects, and pickup of spurious if signals. We show that the temperature coefficient of the new NIST HRl synthesizers is less than $1 \mathrm{ps} / \mathrm{K}$ and that the pulling from spectral purity is less than $3 \times 10^{-20}$ in NIST-7, our primary thermal cesium beam standard. The pulling for slow cesium standards should be lower. We also show that the pulling due to spurious lines in Ramsey standards with narrow line widths can be manipulated to examine spectral pulling. The fractional frequency stability is better than $3 \times 10^{-14} \tau^{-1 / 2}$ for measurement times out to $10^{4} \mathrm{~s}$ and reaches $10^{-16}$ in about 15 minutes in a standard laboratory environment of roughly $+/-0.5 \mathrm{~K}$ without the need of additional thermal regulation.
\end{abstract}

\section{Introduction}

A new generation of frequency standards based on cooled atoms or ions promises to attain long term fractional frequency stability of order $10^{-16}$ to $10^{-17}$. These new standards require high resolution microwave excitation frequencies with low phase modulation (PM) noise, low amplitude modulation (AM) noise, low spurs near the carrier, and also very high phase stability with respect to environmental effects [1-5]. The microwave frequency must somehow be connected to a standard reference frequency that can be communicated to a local time scale and then to the world in a manner that minimizes the errors.

This paper presents our latest approach to the problem of constructing a synthesizer with the features needed to attain a frequency stability of $10^{-17}$ for measurement times of one day and longer References [6-8] address many of the problems associated with high stability synthesis, distribution and measurement. This work indicates that while it may be difficult and require sub-kelvin temperature control to construct a synthesizer and measurement system based on 5 or $10 \mathrm{MHz}$ that is capable of $10^{-17}$ at one day, it should be much easier to attain this performance if the primary phase measurements are made at $100 \mathrm{MHz}$. We therefore have chosen to have our most stable output at $100 \mathrm{MHz}$. Examples of previous approaches are detailed in $[9,10]$.

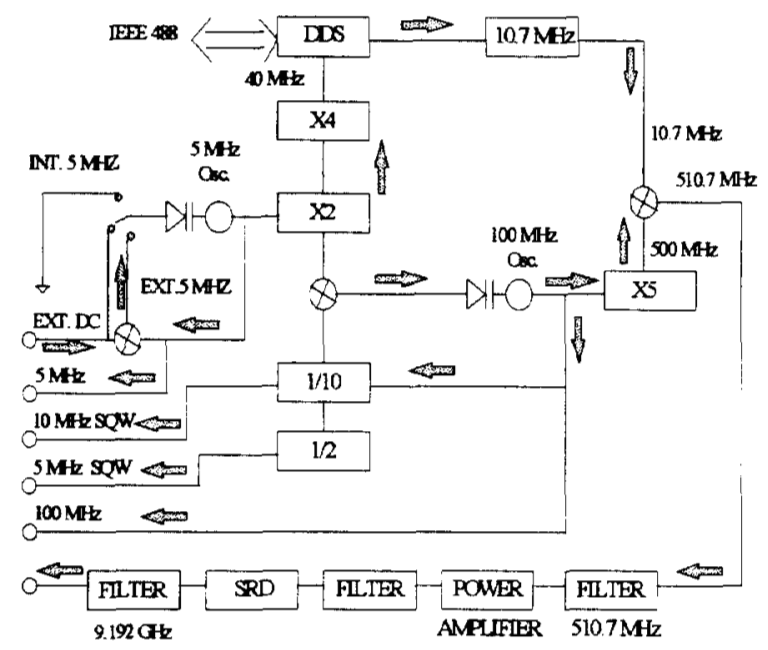

Figure 1. Block diagram of the NIST HR1 series of high stability synthesizers developed at NIST to interrogate passive $\mathbf{R b}$ or $\mathrm{Cs}$ (depicted) atomic frequency standards

\section{Architecture of NIST HR1 Synthesizer}

Figure 1 shows a general block diagram for the high resolution, high stability synthesizer HR1 that can be used to excite Cesium (Cs) (illustrated) or Rubidium $(\mathrm{Rb})$ passive standards. The only change 
needed to interrogate a passive $\mathrm{Rb}$ standard is to select a different offset frequency and associated offset oscillator and filter. The resolution at the offset synthesizer is $1 \times 10^{-6} \mathrm{~Hz}$ leading to a settability of $2 \times 10^{-15}$ for both $\mathrm{Cs}$ and $\mathrm{Rb}$ standards. The primary function of the $5 \mathrm{MHz}$ internal oscillator is to provide good close-in spectral purity and time domain stability for times shorter than about $1 \mathrm{~s}$. The internal/external control permits the unit to be steered by an external dc error signal or an external local oscillator at 5,10 , or $100 \mathrm{MHz}$ with a time constant of order $1 \mathrm{~s}$. The long time constant for the external rf lock is used to exclude spurs due to ground loops and pickup of electromagnetic interference.

The $100 \mathrm{MHz}$ oscillator controls the spectral purity for Fourier offset frequencies larger than about $100 \mathrm{~Hz}$. The output of the $100 \mathrm{MHz}$ oscillator is multiplied to $500 \mathrm{MHz}$ where it is mixed with a modified commercial Direct Digital Synthesizer (DDS) to provide an offset frequency which is an exact submultiple of the desired microwave frequency. The frequency and phase of the offset synthesizer can be adjusted manually or by IEEE 488 control. The offset frequency can be changed over a range of $\pm 10 \mathrm{kHz}( \pm 180 \mathrm{kHz}$ at $\mathrm{Cs}$ frequency) to interrogate Zeeman resonances without exceeding the locking range of the quartz oscillator used to reduce the spurs introduced by the offset synthesizer. An adaptive servo, whose gain increases for large transients, is used to reduce the time needed to attain stable operation after switching the frequency of the offset oscillator [11]. After modification, the effect of noise in the offset DDS on the frequency stability of the output is less than $6 \times 10^{-15}$ at $100 \mathrm{~s}$ and $2 \times 10^{-17}$ at $10^{4} \mathrm{~s}$. The offset $500 \mathrm{MHz}$ signal is then filtered, amplified, and multiplied in a conventional step recovery diode to produce a comb extending to X-band. A band pass filter selects the desired harmonic and reduces the adjacent $500 \mathrm{MHz}$ side bands by approximately $40 \mathrm{~dB}$. The side bands at $1 \mathrm{GHz}$ are less than $-70 \mathrm{dBc}(\mathrm{dB}$ below the carrier).

All synthesizer components, except the commercial DDS offset synthesizer and the power supply, are contained in a shock mounted if and magnetically shielded chassis. This approach yields a very clean microwave spectrum as illustrated in the spectrum analyzer trace shown in Fig. 2 for a Cs synthesizer. Except for the residuals left from filtering the 10.7 and $510.7 \mathrm{MHz}$ comb, there are no spurs higher than $-73 \mathrm{dBc}$

\section{Static Frequency and Phase Stability}

The static frequency and phase stability of a pair of $9.192 \mathrm{GHz}$ synthesizers has been measured by phase locking them together at $9.192 \mathrm{GHz}$ and comparing their phases at the $5 \mathrm{MHz}$ sine wave, $5 \mathrm{MHz}$ square wave, $10 \mathrm{MHz}$ sine wave, and $100 \mathrm{MHz}$ sine wave outputs as shown in Fig. 3. The sensitivity of the phase detector is calibrated by stepping the phase of one of the $10.7 \mathrm{MHz}$ offset synthesizers. The frequency stability of the $5 \mathrm{MHz}$ signals is also measured on our standard measurement system. [12]

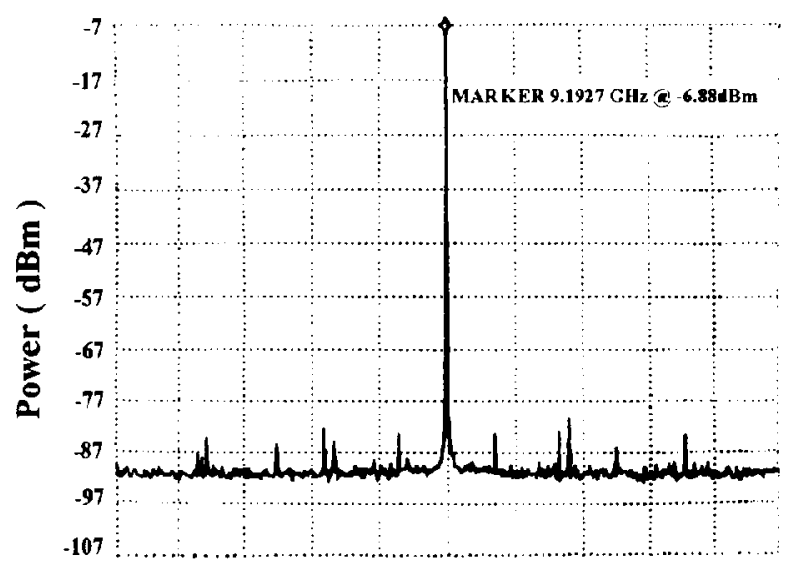

CENTER FREQUENCY $9.19276 \mathrm{GHz}$

SPAN $20 \mathrm{MHz}$ RESOLUTION BANDWIDTH $300 \mathrm{~Hz}$ VIDEOBANDWIDTH $300 \mathrm{~Hz}$

Figure 2. Power spectrum of the $9.192 \mathrm{GHz}$ output of Cs synthesizer HR 1-3

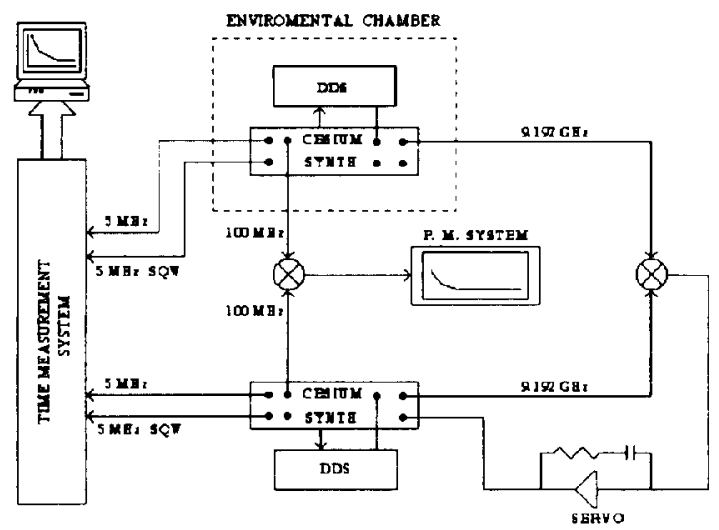

Figure 3. Block diagram for measuring the phase and time domain frequency stability of the various outputs of the HR1 synthesizers.

Figure 4 shows the phase stability over a $8 \mathrm{~h}$ run at $100 \mathrm{MHz}$. One sinthesizer (HR1-3) was held at approximately $\pm 0.3 \mathrm{~K}$ while the temperature of the ther synthesizer followed the room temperature fluctuations of approximately $\mathrm{IK}$. 


\section{TIME ERROR AT 100MHz}

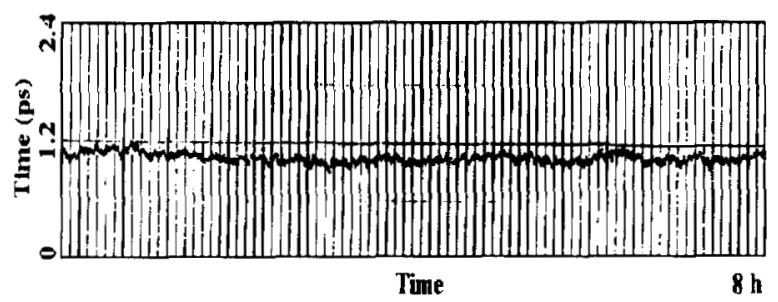

Figure 4. Phase difference between the $100 \mathrm{MHz}$ to $9.192 \mathrm{GHz}$ outputs of HRI-3 versus HRl-4 over an 8 hour period.

Figure 5C shows a time transient of about 3 ps when the temperature of the synthesizer in the chamber was changed by about $6 \mathrm{~K}$ over a $2 \mathrm{~h}$ period. After several hours the phase returned to within $1.2 \mathrm{ps}$ of the original value. This data indicates a temperature coefficient about $0.5 \mathrm{ps} / \mathrm{K}$ for the $100 \mathrm{MHz}$ output of HR1-3. The temperature stability of the $5 \mathrm{MHz}$ sine wave output was approximately $25 \mathrm{ps} / \mathrm{K}$. The temperature stability of the $5 \mathrm{MHz}$ square wave output, obtained by dividing the $100 \mathrm{MHz}$ output, is approximately $10 \mathrm{ps} / \mathrm{K}$. The temperature stability of the $5 \mathrm{MHz}$ square wave output would limit the performance to roughly $\sigma_{\mathrm{y}}(\tau)=1 \times 10^{-16} /(\tau \delta \mathrm{T})$ where $\tau$ is in days and $\delta T$ is the temperature fluctuation in kelvin. Auxiliary tests indicate that the primary contribution to the temperature coefficient of the $5 \mathrm{MHz}$ square wave output is due to the FET buffer amplifier.

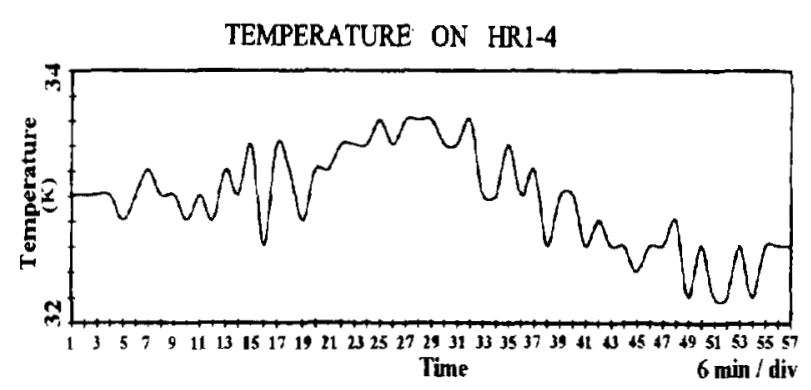

Figure 5A. Temperature profile of synthesizer HR I-4

Figure 6 shows the fractional frequency stability of the $100 \mathrm{MHz}$ output for the pair during the period of approximately $1 \mathrm{~K}$ temperature stability. The fractional stability drops below $10^{-16}$ for measurement times longer than 15 minutes. The temperature stability would limit the performance to roughly $\sigma_{\mathrm{y}}(\tau)=5 \times 10^{-18} /(\tau \delta \mathrm{T})$. Other curves show the fractional frequency stability for the 5 $\mathrm{MHz}$ square wave and $5 \mathrm{MHz}$ sine wave outputs of the synthesizer pair. The approximate resolution of the measurement system was estimated by measuring the same clock in two channels.

TEMPERATURE ON HR1-3

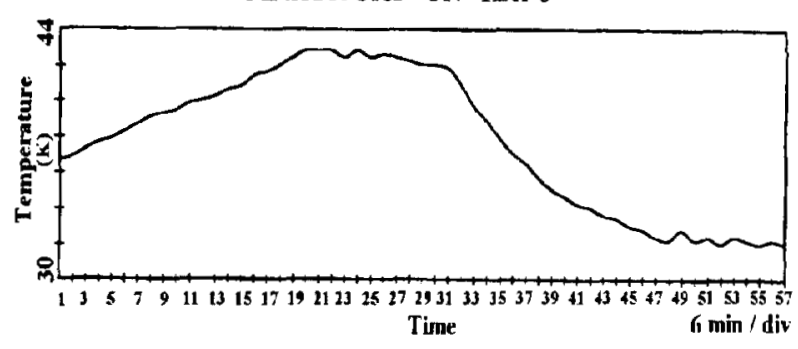

Figure 5B. Temperature profile of synthesizer HR 1-3

TIME STABILITY@ 100 MHz

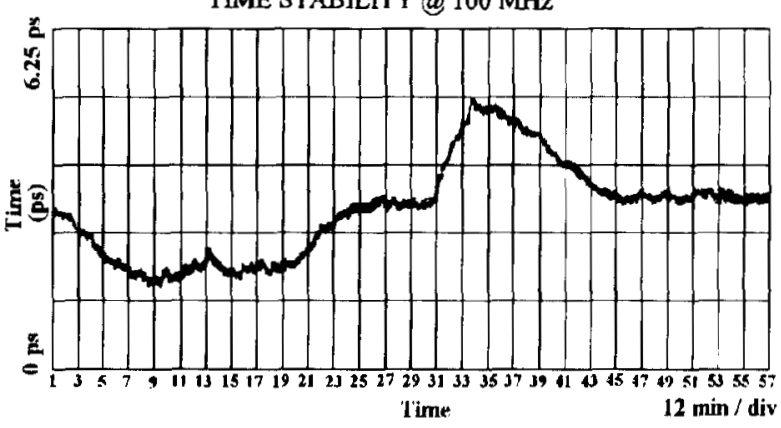

Figure 5C. Phase difference between the $100 \mathrm{MHz}$ to 9.192 GHz outputs of HRl-3 versus HRl-4 for the temperature profiles of $5 \mathrm{~A}$ and $5 \mathrm{~B}$.

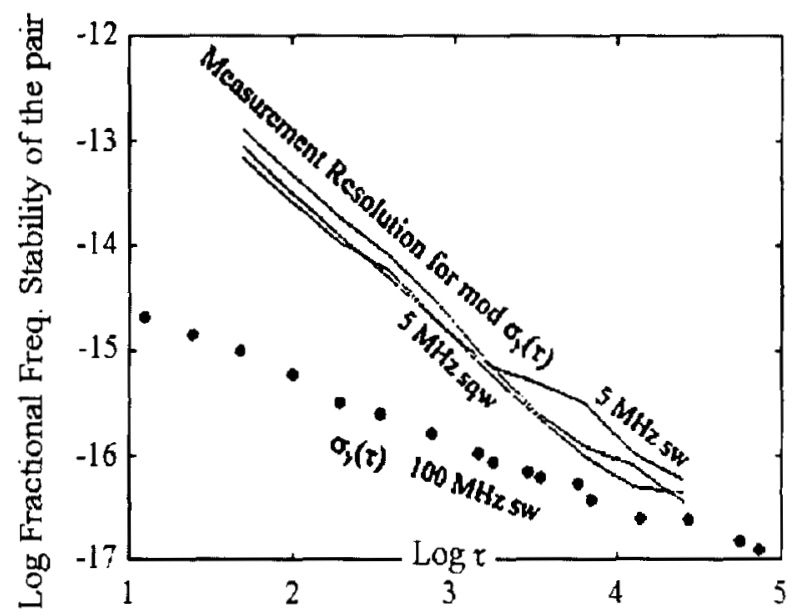

Figure 6. Fractional frequency stability of a pair of HRI Cs synthesizers 


\section{Errors Due to Lack of Spectrum Purity}

Spurious signals can cause significant frequency shifts in high performance frequency standards $[11,13]$. Spurious sideband signals that happen to overlap with the Zeeman or other structure of the atomic resonance can cause frequency pulling by inducing unwanted transitions. The apparent frequency of the resonance is also pulled by nearby spurious sideband signals. For narrow band microwave resonances, only single sideband (SSB) spurs can cause any significant pulling. Figure 7 shows the fractional frequency error induced by a single sideband spur $40 \mathrm{~dB}$ below the carrier for our thermal beam Cs standard, NIST-7, operated at a $2 \mathrm{~dB}$ below optimum microwave power. Experimental verification of the curve for SSB spurs from 0 to $250 \mathrm{~Hz}$ is shown in the inset of Fig. 7 [11]. The peak pulling scales as the line width and inversely as the power $[11,13]$. For NIST-7 the pulling is given by Eq. (1) [11]

$$
\frac{\delta v}{v}=-\frac{\mathrm{K}(\mathrm{SSB})}{10^{-4}},
$$

where $\mathrm{K}$ is given by Fig. 7 and SSB is the power in the single sideband spur.

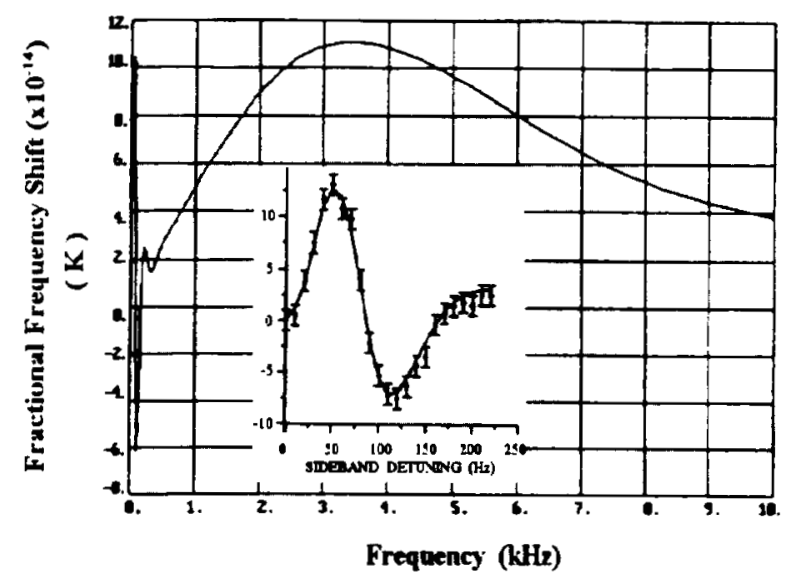

Figure 7. Fractional frequency error due to SSB pulling in a thermal beam Cs frequency standard like NIST-7. The inset shows the experimental confirmation of the theory from [11]

The only way to obtain a SSB spur is to have both a PM and AM spur at exactly the same frequency. The amplitude of this SSB spur can not be larger than twice the amplitude of the single sideband AM or PM spur. Figure 8 shows the PM noise and the PM spurs from $1 \mathrm{~Hz}$ to $100 \mathrm{kHz}$ for the pair of synthesizers. We changed the offset synthesizers up to $8 \mathrm{~Hz}$ and saw no difference in the amplitude of the PM spurs [10].

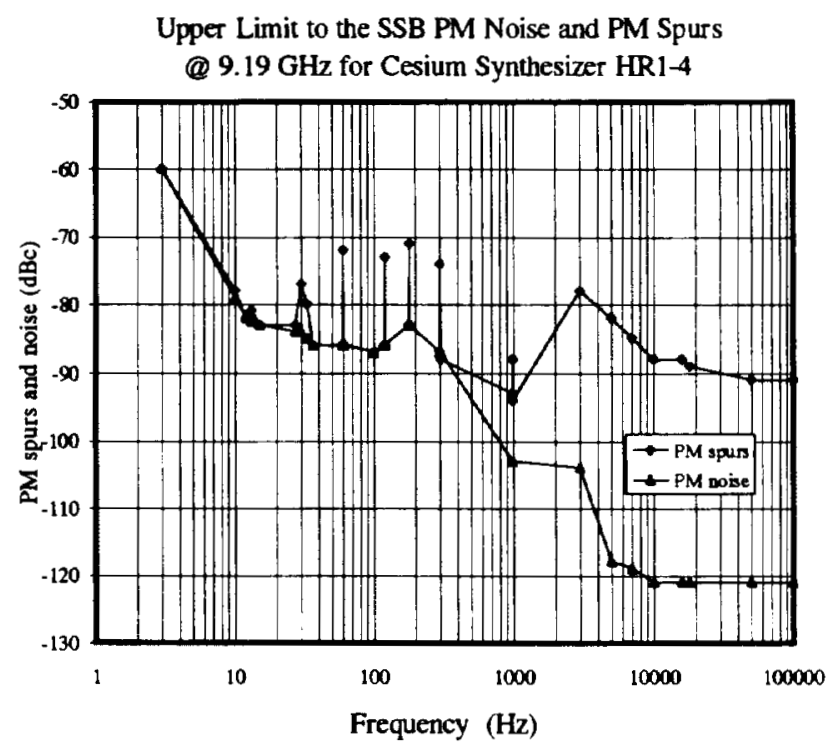

Figure 8. The typical PM noise and upper limit to the PM spurs for the Cs version of our HR1 synthesizers

Upper Limit to the SSB AM Noise and AM Spurs (1) 9.19 GHz for Cesium Synthesizer HR1-4

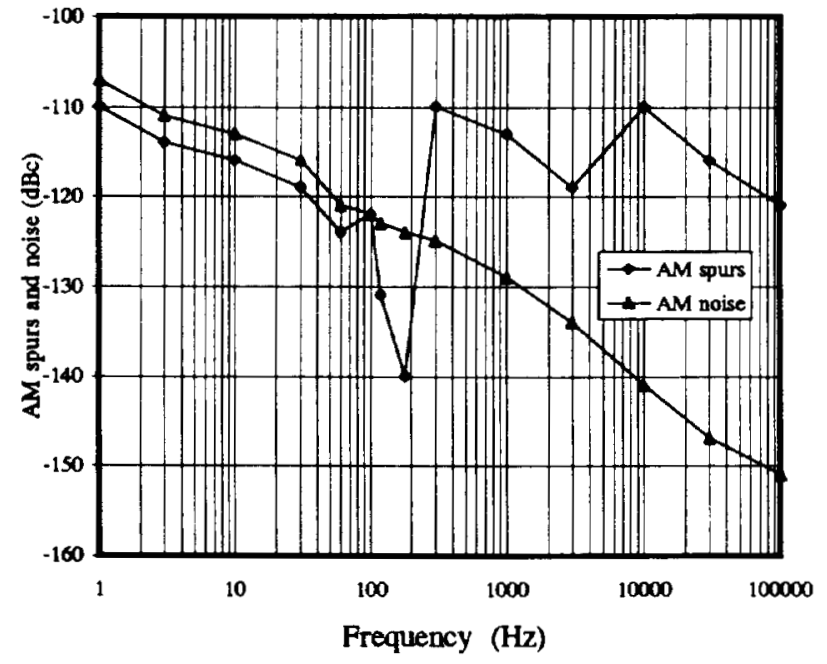

Figure 9. The typical AM noise and upper limit to the AM spurs for the Cs version of our HRI synthesizers

Figure 9 shows the upper limit to the AM noise and the limit to the AM spurs for Fourier offset frequencies from $1 \mathrm{~Hz}$ to $100 \mathrm{kHz}$. The values for the AM spurs at 60,120 and $180 \mathrm{~Hz}$ were obtained by narrow band measurements. The results of Fig. 9 combined with those of Fig. 7 and Equation (1) show 
that SSB spurs in our synthesizer cause frequency errors of less than $2 \times 10^{-20}$ in NIST-7.

The PM spurs at 13 and $29 \mathrm{~Hz}$ are very interesting because they are due to vibration of the building enhanced by resonances in the bench and mounting structure. These spurs disappear when the synthesizer is mounted on a more rigid structure or the orientation of the synthesizer is changed. Figure 10 shows the vibration measured on the floor of the laboratory, a bench top and the top of a HRl synthesizer. The peaks at 13 and $29 \mathrm{~Hz}$ correspond to normal modes of the building excited by large mechanical equipment used for controlling the building environment. The spurs at 58 and $97 \mathrm{~Hz}$ are generated by fans in various pieces of electronic test equipment. Figure 11 shows the influence in the PM noise of the synthesizer at a carrier frequency of $100 \mathrm{MHz}$. From Figs. 10 and 11 we calculate an acceleration sensitivity of $2 \times 10^{-9} / \mathrm{g}$ acceleration. These PM spurs have no effect on the frequency pulling because there are no corresponding AM spurs at the same frequencies. We were not able to observe any affect on the spur structure due to acoustic noise.

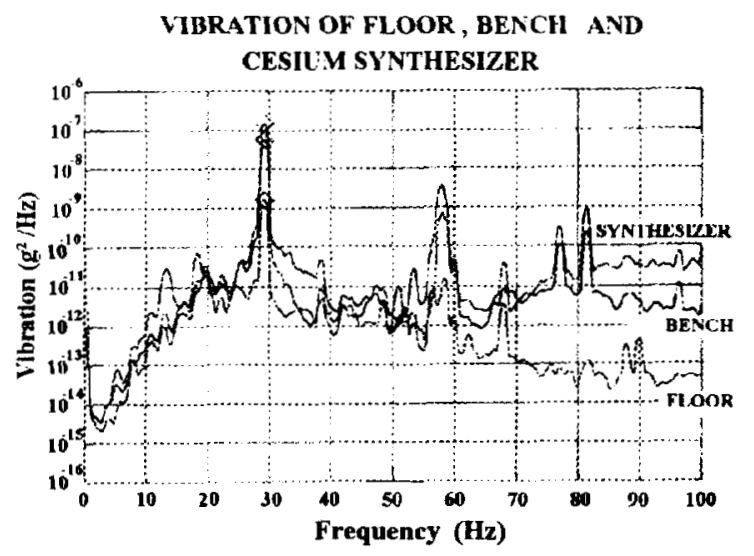

Figure 10. Vibration spectrum of the floor of the laboratory, a bench, and a HRI synthesizer placed on the bench.

The frequency offset of a typical fountain Cs standard would be much less since the linewidths are roughly a factor of 50 times smaller and hence the pulling factor $\mathrm{K}$ would roughly be 50 times smaller. The approximate peak values and envelope of the $K$ factor for a typical fountain are shown in Fig. 12. The width and spacing of the oscillating lines (but not the envelope) has been increased by about a factor of 3 for clarity.

An interesting aspect of frequency standards which use Ramsey interrogation is that it is possible to significantly change the frequency shift due to spurious signals at Fourier frequency components larger than the line width. Slow Cs frequency standards have a line width of about $1 \mathrm{~Hz}$ while that of the trapped ion frequency standards is typically less than $0.01 \mathrm{~Hz}$. In contrast to NIST-7 with its thermal beam, the frequency pulling in standards with slow atomic resonators exhibits a comb like structure that is about the same line width as the central Ramsey line. This structure extends to Fourier frequencies of about the Rabi line width and then falls as $1 / f$ as illustrated by Fig. 12. By adjusting the Rabi time or the Ramsey time by a small amount, one can change the position of the peaks and valleys of the frequency pulling to correspond to the frequency of the potential spurious side bands. This feature should prove very powerful in evaluating these effects in both stored ion and fountain type frequency standards.

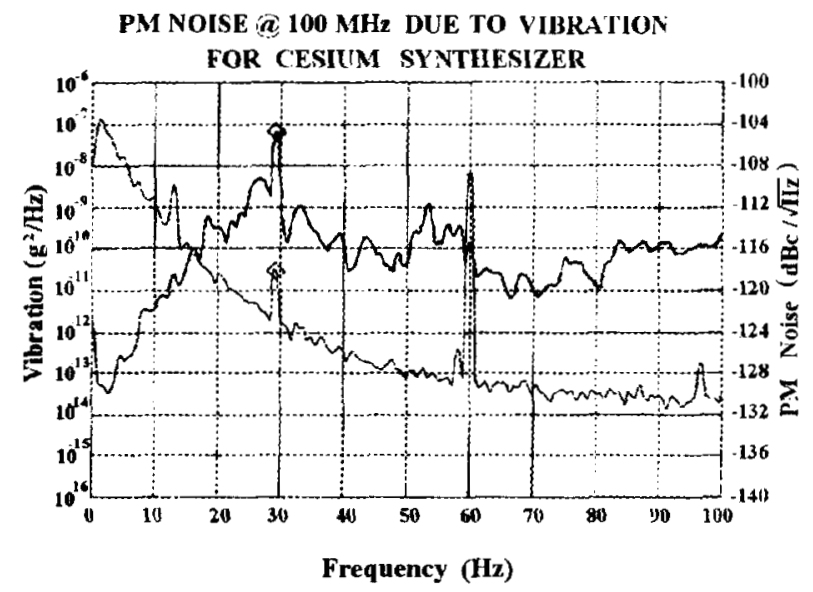

Figure 11. PM spurs at $100 \mathrm{MHz}$ in a HRI type synthesizer due to the vibration levels of Fig. 10

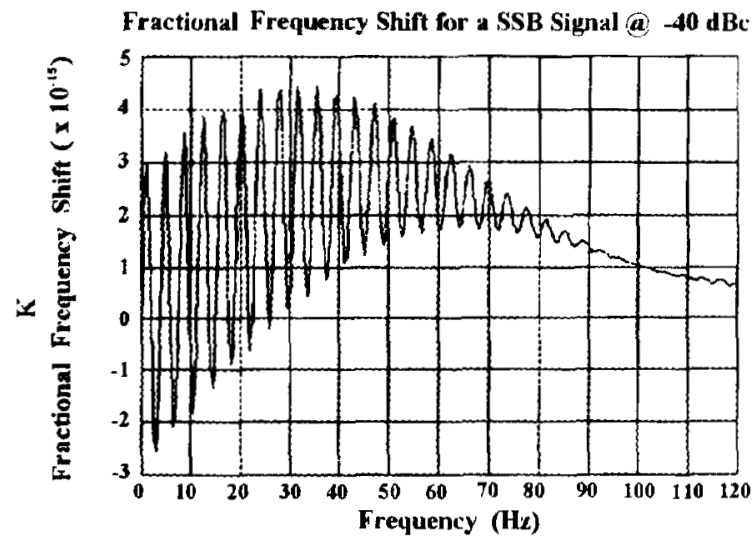

Figure 12. Envelope of the estimated fractional frequency error due to SSB pulling in a slow Cs fountain type frequency standard. The spacing of the oscillatory structure has been increased by a factor of 3-4 for clarity. 
5MHz Spurs on the Power Line

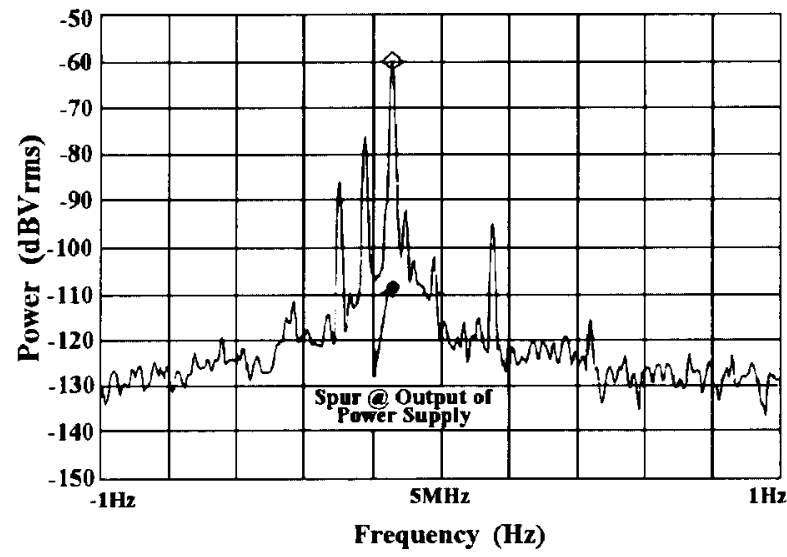

Figure 13. Spurious noise on the power line in our laboratory near $5 \mathrm{MHz}$. Also indicated is the upper limit to the $5 \mathrm{MHz}$ spurs at the output of the shielded power supplies for HR1 type synthesizers.

Figure 13 shows the spurious signals on the $60 \mathrm{~Hz}$ power line near $5 \mathrm{MHz}$. The highest spur is $-60 \mathrm{dBV}$ ( $\mathrm{dB}$ below 1V). The shielded power supply reduces this to at least $-110 \mathrm{dBV}$. Each if or microwave module is individually regulated and filtered to reduce these signals even further. If one assumes that a spurious signal of $-110 \mathrm{dBV}$ at $5 \mathrm{MHz}$ is injected into the normal $+10 \mathrm{dBV} 5 \mathrm{MHz}$ signal path, the resulting phase modulation would be $10^{-6}$ radians at $5 \mathrm{MHz}$ and $2 \times 10^{-3} \mathrm{rad}$ at $9.192 \mathrm{GHz}$. This corresponds to a time modulation of $3 \times 10^{-14} \mathrm{~s}$. The actual modulation due to this effect is probably much smaller than this worse case estimate.

\section{Conclusion}

We have described the architecture and philosophy of the NIST HRl high stability high resolution synthesizer designed to probe passive $\mathrm{Cs}$ or $\mathrm{Rb}$ frequency standards. This synthesizer can be stepped $\pm 180 \mathrm{kHz}$ at $9.192 \mathrm{GHz}$ to interrogate Zeeman resonance and has a frequency resolution of $2 \times 10^{-15}$. We have evaluated the frequency and phase stability of a pair of these synthesizers and find a temperature coefficient of less than $1 \mathrm{ps} / \mathrm{K}$ for synthesis from $100 \mathrm{MHz}$ to $9.192 \mathrm{GHz}$ and $10 \mathrm{ps} / \mathrm{K}$ for synthesis from $5 \mathrm{MHz}$ to $9.192 \mathrm{GHz}$. The typical frequency stability of the $100 \mathrm{MHz}$ to $9.192 \mathrm{GHz}$ output is approximately $1 \times 10^{-16}$ at 15 minutes and approaches $1 \times 10^{-17}$ at a day in a laboratory with thermal variations of about $\mathrm{l} \mathrm{K}$ without the need for any additional temperature regulation. The spectral purity is excellent, with all spurs more than $-73 \mathrm{~dB}$ below the carrier $(\mathrm{dBc})$ for Fourier frequencies from 1 to $100 \mathrm{kHz}$. SSB spurs are less than $-110 \mathrm{dBc}$ over the same span. The SSB spurs are so low that the pulling on the atomic resonance is less than $2 \times 10^{-20}$ for NIST-7 and even lower for a typical fountain type Cs frequency standard. We also show that vibration effects are about $2 \times 10^{-9} / \mathrm{g}$ and have little effect on the synthesizer performance if the unit is mounted on a rigid structure. The effects of spurious rf signals on the power lines are negligible.

\section{$\underline{\text { References }}$}

[1.] D.J. Wineland, J.C. Bergquist, J. Bollinger, W.M. Itano, D.J. Heinzen, S.L. Gilbert, C.H. Maney, and M.G. Raizen, "Progress at NIST Towards Absolute Frequency Standards using Stored Ions," IEEE Trans UFFC 37, pp 515-523, 1990.

[2.] R.L. Tjoelker, J.D. Prestage, G.J. Dick, and L. Maleki, "Long Term Stability of $\mathrm{Hg}^{+}$Trapped Ion Frequency Standard," Proc. 47th Ann. Symp. Freq. Cont. pp 132-138, 1993.

[3.] A. Clairon, P. Laurent, G. Santarelli, S. Ghezali, S.N. Lea, and M. Bahoura, "A Cesium Fountain Frequency Standard: Preliminary results," IEEE Trans. I\&M, IM-44, pp 128-131, 1995.

[4.] J.D. Miller, D.J. Berkland, F.C. Cruz, J.C. Bergquist, W.M. Itano, and D.J. Wineland, "A LaserMicrowave Double Resonance Frequency Standard," to be published in the Proc. 1996 IEEE Int. Freq. Cont. Symp. 1996.

[5.] G.J. Dick, "Local Oscillator Induced Instabilities in Trapped Ion Frequency Standards," Proc. 19th Ann. PTTI, pp 133-147, 1987.

[6] F. L. Walls, L.M. Nelson, and G.R. Valdez, "Designing at the $10^{-18}$ level," Proc. 6th EFTF pp 477481, 1992.

[7] L.M. Nelson and F..L. Walls, "Environmental Effects In Mixers and Frequency Distribution Systems," Proc. of 1992 IEEE Freq. Cont. Symp., pp 831-837, May. 1992.

[8]. F.L. Walls and F. G. Ascarunz, "The Effect of Harmonic Distortion on Phase Errors in Frequency Distribution and Synthesis," Proc. 9th EFTF, pp 170$176,1995$.

[9] C.W. Nelson, F.L. Walls, F.G. Ascarrunz, and P.A. Pond, "Progress on Prototype Synthesizer Electronics for $199 \mathrm{Hg}^{+}$at $40.5 \mathrm{GHz}$," Proc. 1992 IEEE Freq. Cont. Symp., pp 64-69, 1992.

[10] G.D. Rovera, G. Santarelli, and A. Clairon, "Frequency Synthesis Chain for the Atomic Fountain 
Primary Frequency Standard," Proc. IEEE Trans. UFFC 43, pp 354-358, 1996.

[11] W.D. Lee, J.H. Shirley, F.L. Walls, and R.E. Drullinger, "Systematic Errors in Cesium Beam Frequency Standards Introduced by Digital Control of the Microwave Excitation," Proc. 1995 Int. Freq. Cont. Symp. pp 113-117.

[12] S.R. Stein, D. Glaze, J. Levine, D. Hilliard, D. Howe, and L. Erb, "Automated High Accuracy Phase Measurement System," IEEE Trans. I\&M IM-32, pp 227-231, 1983

[13] C. Audoin, M. Jardino, L.S. Cutler, and R.F. Lacy, "Frequency Offset due to Spectral Impurities in Cesium-Beam Frequency Standards," IEEE Trans. I\&M. IM-27, pp 325-329, 1978. 